\title{
GLOBAL STABILITY ANALYSIS - A KEY ENABLER IN REDUCED ORDER MODELS AND FLOW CONTROL
}

\author{
M. Morzyński ${ }^{1}$, W. Stankiewicz ${ }^{1}$, F. Thiele ${ }^{2}$, B. R. Noack $^{2}$, \\ and G. Tadmor ${ }^{3}$ \\ ${ }^{1}$ Poznań University of Technology \\ Institute of Combustion Engines and Transportation \\ Poland \\ ${ }^{2}$ Institut Pprime, CNRS - Université de Poitiers - ENSMA \\ Département Fluides, Thermique, Combustion, CEAT \\ Poitiers, France \\ ${ }^{3}$ Department of Electrical and Computer Engineering \\ Northeastern University \\ Boston, USA
}

In the current study, Reduced Order Models (ROMs) targeting strategies for experimental feedback flow control are discussed. For practical reasons, such models should incorporate a range of flow operating conditions with a small number of degrees of freedom. Standard POD Galerkin models are challenged by overoptimization at one operating condition [1]. The extension of dynamic range with additional global flow stability modes is the first applied technique. Further side constraints for control-oriented ROMs are taken into account by a 'least-dimensional' Galerkin approximation based on a novel technique for continuous mode interpolation [2]. This interpolation preserves the model dimension of a single state while covering several states by adjusting (interpolated) modes. The resulting three-dimensional (3D) Galerkin model is presented for the transient flow around NACA-0012 airfoil and shown to be in a good agreement with the corresponding direct numerical simulation (DNS).

\section{INTRODUCTION}

Computational Fluid Dynamics (CFD) is a mature tool used in design and improvement of performance of transport systems such as airplanes, trains, or cars.

This is an Open Access article distributed under the terms of the Creative Commons Attribution-Noncommercial License 3.0, which permits unrestricted use, distribution, and reproduction in any noncommercial medium, provided the original work is properly cited. 
Development of high fidelity CFD systems is accompanied by the clear conclusion that parametric studies cannot rely only on increasing computer power or even parallelization [3]. The design process opens myriads of versions to be analyzed. Low fidelity analysis and/or ROMs are presently the only realistic alternative. Reduced order models are also necessary in the closed-loop flow control. Modelbased flow control requires online-capable feedback laws. In this paper, a focus on a system reduction and the use of global flow stability eigenmodes as the key strategy to improve the flow model dynamics is made. Traditionally, Proper Orthogonal Decomposition (POD) modes, being the result of pure signal processing, were used for ROMs. Poor performance of the models built with this basis triggered several novel ideas and improvements [1, 4-10]. The successful approaches incorporated more physical information about the modeled system. The use of stability eigenmodes and continuous mode interpolation presented in this paper is an example of this strategy.

\section{EMPIRICAL GALERKIN MODEL}

The standard Galerkin method [11] decomposes the velocity field into a base flow $\mathbf{u}_{0}$ and fluctuation $\mathbf{u}^{\prime}$. The velocity field is approximated in the physical domain $\Omega$ with the space-dependent expansion modes $\mathbf{u}_{i}$ and time-dependent Fourier coefficients $a_{j}$ :

$$
\mathbf{u}^{[0 . . N]}=\mathbf{u}_{0}+\sum_{j=1}^{N} a_{j} \mathbf{u}_{j}, \quad a_{0} \equiv 1 .
$$

The ansatz (1) can be used to derive a high-dimensional Finite Element model (FEM) (computational Galerkin method) if expansion modes have local compact support on grid cells (FEM hats). Low dimensionality and robustness, which is our goal in designing the flow model, requires a traditional Galerkin method that is based on global expansion modes. The Galerkin system, resulting from projection of the Navier-Stokes equations onto the space spanned by the expansion modes has the form:

$$
\frac{d}{d t} a_{i}=\frac{1}{\operatorname{Re}}>\sum_{j=0}^{N} l_{i j} a_{j}+\sum_{j, k=0}^{N} q_{i j k} a_{j} a_{k}
$$

where

$$
l_{i j}=\left(\mathbf{u}_{i}, \triangle \mathbf{u}_{j}\right)_{\Omega} ; \quad q_{i j k}=-\left(\mathbf{u}_{i}, \nabla \cdot\left(\mathbf{u}_{j} \otimes \mathbf{u}_{k}\right)\right)_{\Omega} .
$$

The pressure term may be neglected in the case of absolutely unstable wake flows and arbitrarily large domains [12]. Equation (2) is a low-dimensional analogue of DNS. The Reynolds-averaged Navier-Stokes (RANS) equivalent form 
together with Finite Time Thermodynamic (FTT) closure is described in detail in $[13]$.

\section{GLOBAL FLOW STABILITY EIGENMODES}

The incompressible Navier-Stokes equation

$$
\dot{u}_{i}+u_{i, j} u_{j}+p_{, i}-\frac{1}{R e} u_{i, j j}=0
$$

linearized for small disturbances, with the exponential anzatz for time dependence, yields the generalized complex eigenvalue problem:
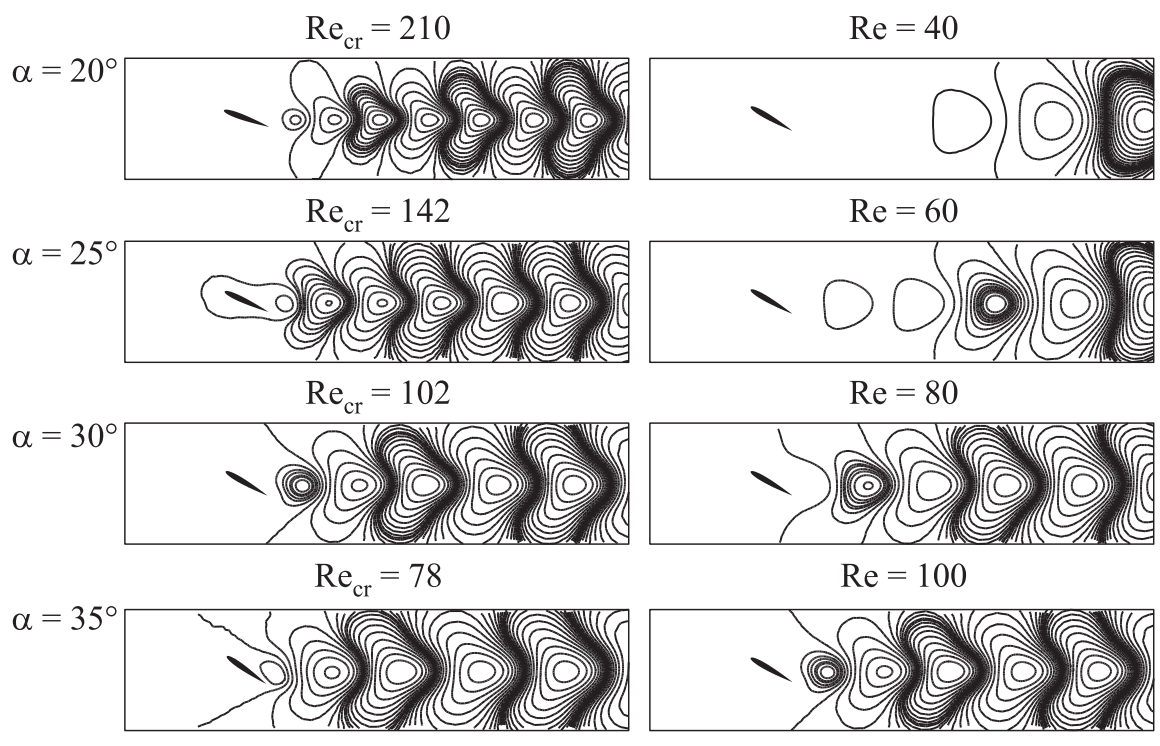

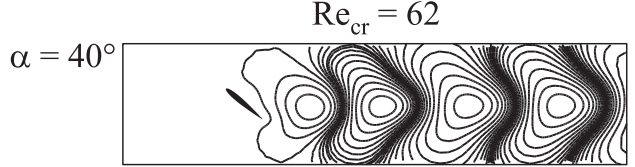

(a)

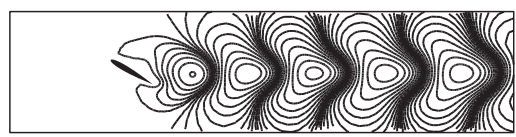

(b)

Figure 1 Streamlines of the most unstable modes corresponding to critical Reynolds numbers at different angles of attack $(a)$; and the evolution of the most unstable eigenmodes with the growth of Reynolds number for $\alpha=30^{\circ}(b)$. Streamlines are visualized in the figure 


$$
\lambda \tilde{u}_{i}+\tilde{u}_{j} \bar{u}_{i, j}+\bar{u}_{j} \tilde{u}_{i, j}+\tilde{p}_{, i}-\frac{1}{\operatorname{Re}} \tilde{u}_{i, j j}=0 ; \quad \tilde{u}_{i, i}=0 .
$$

After discretization, Eq. (3) takes the form:

$$
A x-\lambda B x=0
$$

characterized by a very large dimension of the weakly-conditioned unsymmetrical matrices. Several papers deal with the solution of global flow stability problems, to mention, for example, the recent ones [14-16] and the review given in [17]. In [18], the solution of a large global stability eigenvalue problem with the unstructured 3D FEM is discussed.

Stability analysis is traditionally a tool for prediction of amplification or damping of external disturbances present in all real flows. Usually, this method delivers two kinds of information: the physical modes (eigenmodes) being the form of (spatial) disturbance development and eigenvalues, where the real and imaginary parts are a measure of periodicity in time and amplification of the flow structure - the growth rate.

The physical modes are of particular interest for flow modeling. In Fig. 1, the real part of the eigenvector is shown for the flow around NACA-0012 airfoil at different flow conditions.

\section{IMPROVEMENTS OF THE GALERKIN MODEL}

\subsection{Mean-Field Correction}

The ROM obtained with the POD Galerkin method is highly efficient and resolves the kinematics of the flow nearly perfectly. At the same time, it is highly fragile and sensitive to changes in the parameters or operating conditions. The first two POD modes capture about $95 \%$ of the perturbation energy; yet, the Galerkin model based on these two modes is structurally unstable. The inclusion of eight POD modes, capturing the first four harmonics of the attractor, suffices to achieve nearly perfect resolution and a structurally stable Galerkin system. Yet, the correct prediction of the dynamics of the system with this model is limited to a small neighborhood of the attractor and to relatively small Reynolds number perturbations. Stabilization of the Galerkin model can be obtained with the shift mode [19] as suggested by mean-field theory. The shift mode is a normalized difference $\mathbf{u}_{0}-\mathbf{u}_{s}$ where $\mathbf{u}_{0}$ is the mean flow solution and $\mathbf{u}_{s}$ is the (unstable) steady solution.

The inclusion of the shift mode reduces model sensitivity to parameter variations and is an enabler for the low-dimensional representation of transient manifolds, such as the one connecting the unstable steady solution to the attractor. 


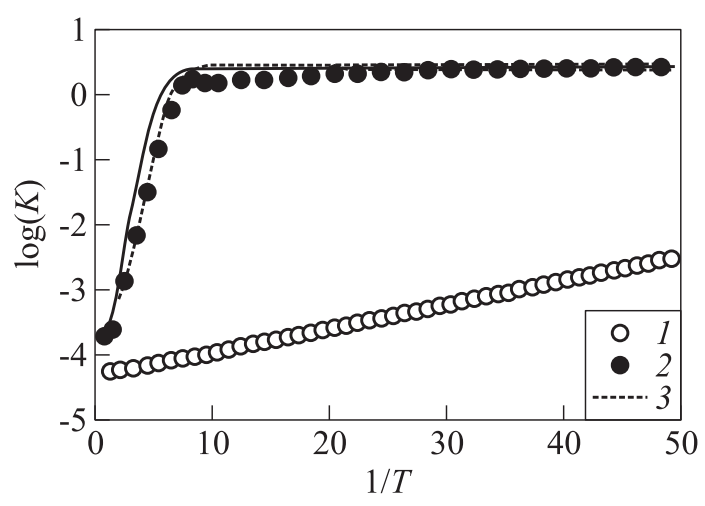

Figure 2 Transients of Galerkin models for the flow around a circular cylinder; 1 denotes traditional, 8 POD mode Galerkin model improvement due to the hybrid model (2) and continuous mode interpolation (3 - two interpolated modes) is seen

The dynamics of the minimum Galerkin Model with a shift mode is compared with DNS in Fig. 2. The shift mode is the key enabler for construction of transient, control-oriented models.

\subsection{Hybrid Model Employing Stability Modes}

Further improvement of the model dynamics is obtained with the hybrid model employing stability modes [20]. In this model, POD modes resolve the attractor and the stability eigenmodes resolve the linearized dynamics. Thus, dynamic transient and posttransient flow behavior is accurately predicted. The concept of the hybrid model reduces significantly the number of necessary degrees of freedom of the system. This approach is demonstrated for the benchmark problem of the flow around circular cylinder in [19]. The transients of the hybrid models are compared with DNS in Fig. 2. The hybrid model combines the advantages of both reduced models. It converges to the limit cycle preserving initially the growth rate predicted by the global stability analysis.

\subsection{Continuous Mode Interpolation}

Further improvement in the design of the least-dimensional ROM flow model is the continuous mode interpolation technique applied for circular cylinder flow in [2]. The mode interpolation smoothly connects not only different operating conditions, but also stability and POD modes (Fig. 3). 


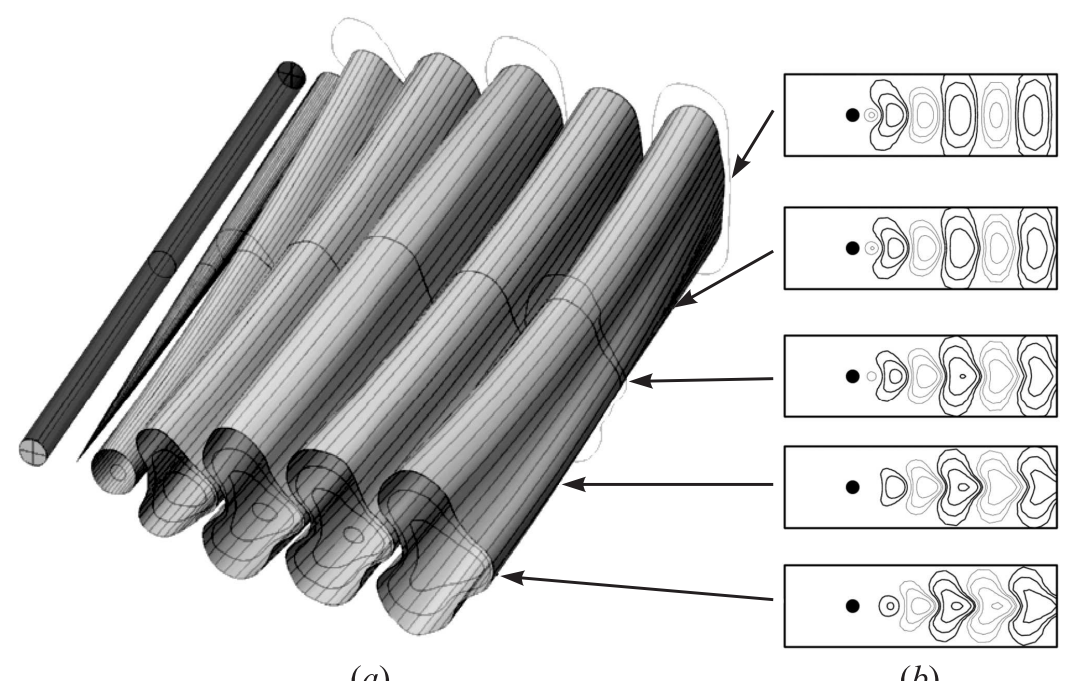

(a)

(b)

Figure 3 Principle sketch of continuous mode interpolation: $(a)$ transition between stability eigenmodes and POD modes; and $(b)$ streamlines of the intermediate states

In this technique, two eigenproblems $A^{0}$ and $A^{1}$, representing the terminal states, are linearly interpolated in $\kappa \in[0,1]$ :

$$
A^{\kappa}=A^{0}+\kappa\left(A^{1}-A^{0}\right) .
$$

In the case of POD modes, the matrices represent discretized Fredholm kernels (autocorrelation function)

$$
\mathbf{A}^{\kappa}(x, y)=\mathbf{u}_{1}^{\kappa}(x) \otimes \mathbf{u}_{1}^{\kappa}(y)+\mathbf{u}_{2}^{\kappa}(x) \otimes \mathbf{u}_{2}^{\kappa}(y)+\ldots
$$

of a Fredholm eigenproblem in the space domain:

$$
\int_{\Omega} \mathbf{A}(x, y) \mathbf{u}_{i}(y) d y=\lambda_{i} \mathbf{u}_{i}(x) .
$$

In the case of eigenmode interpolation, the matrices representing linearized Navier-Stokes equations are utilized.

The eigenvectors of the interpolated eigenproblem (interpolated modes) $\mathbf{u}^{\kappa}$ can be used to model all the intermediate states between $\kappa=0$ and 1 . In addition, the extrapolation of modes outside the design conditions is possible.

Interpolated modes enable 'least-order' Galerkin models, keeping the dimension from a single operating condition but resolving several states: 


$$
\dot{a}_{i}^{\kappa}=\frac{1}{\operatorname{Re}} \sum_{j=0}^{N} l_{i j}^{\kappa} a_{j}^{\kappa}+\sum_{j=0}^{N} \sum_{k=0}^{N} q_{i j k}^{\kappa} a_{j}^{\kappa} a_{k}^{\kappa}
$$

where

$$
\dot{\kappa}=F\left(\kappa, \mathbf{a}^{\kappa}, t\right), \quad \mathbf{a}^{\kappa}:=\left(a_{1}^{\kappa}, a_{2}^{\kappa}, \ldots, a_{N}^{\kappa}\right) .
$$

These models are especially well suited for control design. The results showing transients of circular cylinder flow modeled with the use of continuous mode interpolation are depicted in Fig. 2.

\section{LEAST-ORDER GALERKIN MODEL OF NACA-0012 AIRFOIL FLOW}

The technique presented in the previous section is applied here for the laminar flow around a NACA-0012 airfoil (Fig. 4). More technical details of the approach can be found in [21].

In the present study, a number of different mode bases are considered in the construction of ROMs.

Empirical models use POD modes (Fig. 5) computed with the snapshot technique of Sirovich [22]. Here, the models based on the two and eight most energetic POD modes are analyzed.

Another flow model is designed with the stability eigenmodes, computed using steady and time-averaged solutions as a base flow (Fig. 6).

The last of the models presented in this paper is the least-dimensional model of two modes and continuous mode interpolation. The mean-field correction (shift mode) is employed for all models presented here to avoid the structural instability and fragility. The comparison of all models is shown in Figs. 7-9.

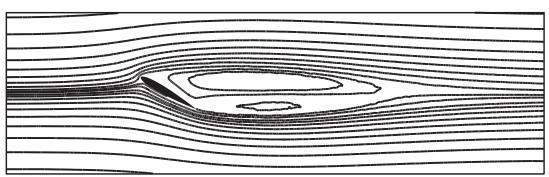

(a)

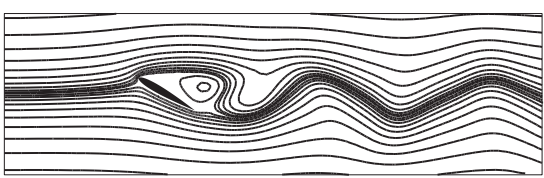

(b)

Figure 4 Flow around a NACA-0012 airfoil: $(a)$ streamlines of the steady solution; and $(b)$ streamlines of the snapshot from the periodic (limit-cycle) flow 


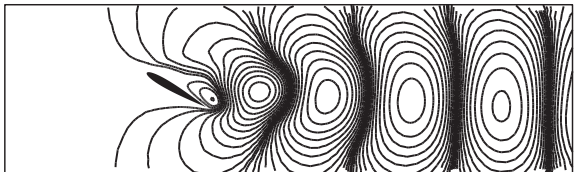

(a)

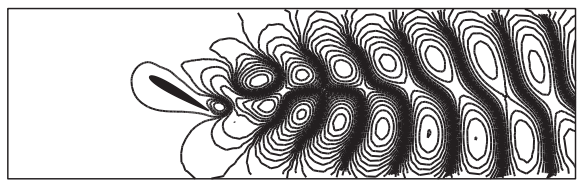

(c)

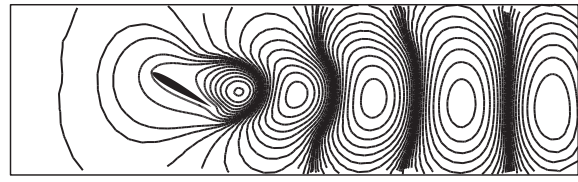

(b)

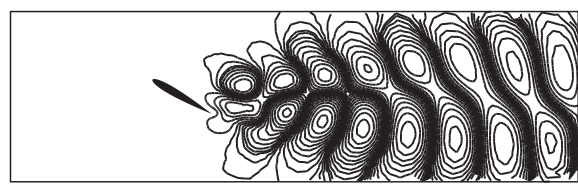

(d)

Figure 5 First 4 POD modes used in Galerkin modeling
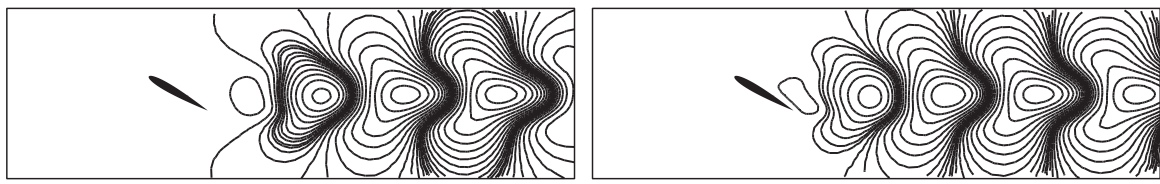

(a)

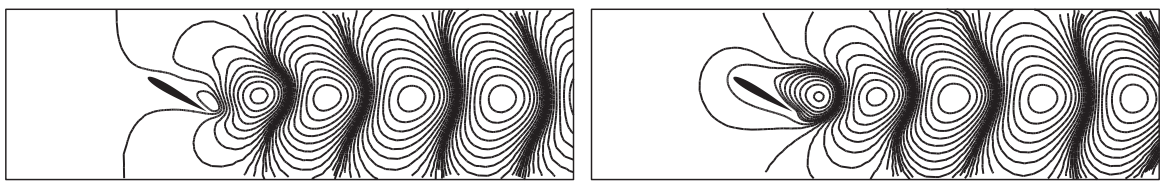

(b)

Figure 6 The most dominant eigenmodes based on the steady solution $(a)(\lambda$ $=-0.147 \pm 0.720 \imath)$ and time-averaged solution $(b)(\lambda=0.018 \pm 0.915 \imath)$. The right and left figures are the real and imaginary parts of the eigenvectors, respectively

It can be seen in Fig. 7 that POD modes allow the reconstruction of the Navier-Stokes attractor (limit cycle), but they are unable to reproduce the dynamical properties of the transitional flow. For the flow states close to the fixed point (steady solution, small values of the shift-mode coefficient), the kinetic energy of the flow is overestimated - especially, in the POD-2 Galerkin model.

The models based on the two most unstable eigenmodes (Fig. 9b) reconstruct the flow states close to the fixed-point (steady solution) and the transition to the limit cycle better than POD Galerkin models. On the other hand, the limit-cycle turbulence kinetic energy (TKE) and shift-mode coefficients of the periodic flow are significantly underestimated with these mode bases. 


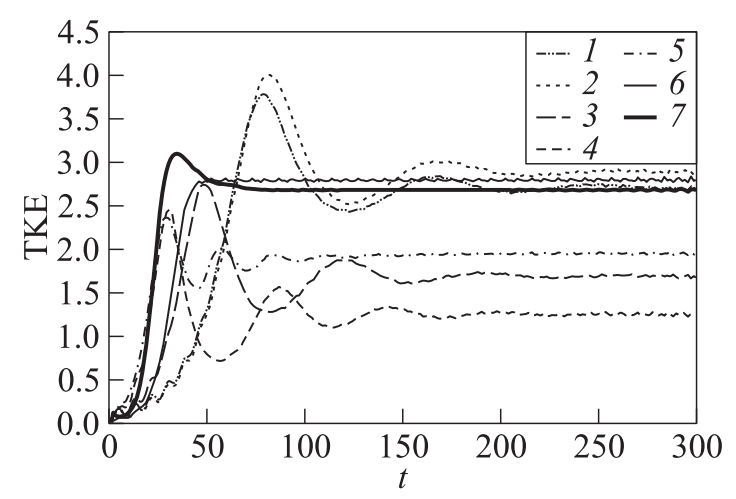

Figure 7 The variation of TKE for different Galerkin models: 1 - POD-8; $2-$ POD-2; 3 - EigM-2; 4 - EigS-2; 5 - Hybrid-2-2; and 6- Interpolated - compared with DNS (7) as a function of time for transition from steady state to the limit cycle oscillation

To take the advantage of both mode bases, an interpolated model of the flow around a NACA-0012 airfoil is used.

To parameterize the transition from the unstable fixed-point (steady solution) to the stable (limit-cycle oscillations) attractor, the interpolation parameter $\kappa$ is related to the shift-mode amplitude (coefficient); $\kappa=0$ represents the steady state and dynamics described by the most stable stability eigenmodes, while $\kappa=1$ is related to the limit-cycle dynamics and POD modes.

Such a model preserves low dimensionality (4 equations for the shift-mode amplitude, interpolated mode amplitudes, and $\kappa$ ) and provides high accuracy in a wide range of operating conditions.

\section{CONCLUDING REMARKS}

In the present paper, the authors emphasized the necessity of ROMs of flows in closed loop flow control as well as in the design process. Reduced order 


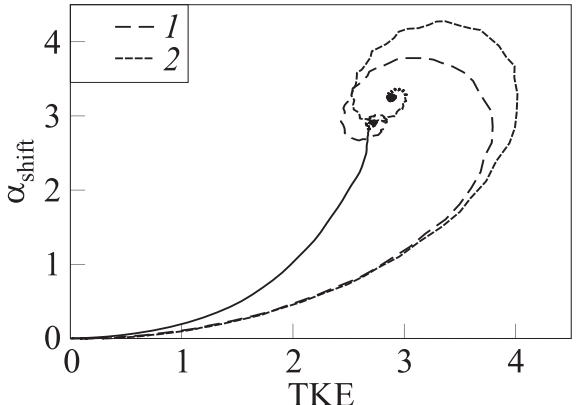

(a)

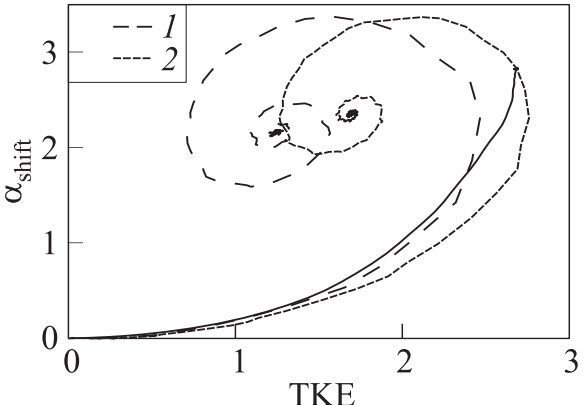

(b)

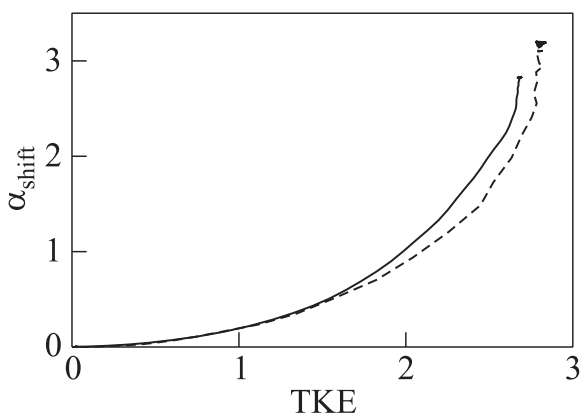

(c)

Figure 9 Shift-mode coefficient as a function of TKE for POD-based models $(a)$ $(1$ - POD-8 and 2 - POD-2), eigenmode-based models $(b)(1-$ EigS-2 and 2 EigM-2), and the interpolated model $(c)$. Solid curves refer to DNS

model is able to deliver technically relevant answers in the fraction of the time necessary for the full scale computations. Robust ROM of the flow is also the most important element of online-capable feedback flow control.

The authors concentrate here on assuring the adequate dynamical properties of the model. Traditional methods of ROM construction, based on the assembly of POD modes, are dynamically fragile and overoptimized at single operating conditions. Enrichment of the mode basis is one of the key techniques of improvement. The shift mode, providing the missing direction from the fixed point to the limit cycle assures the convergence of the Reduced Order Galerkin Model. Hybrid models, employing stability eigenmodes further improve the dynamics. The construction of the least-dimensional flow model, preserving accuracy in a wide range of operating conditions, is possible with the continuous mode interpolation between POD and stability eigenmodes.

A key enabler in ROMs presented here and in flow control is the global stability analysis. 


\section{ACKNOWLEDGMENTS}

The authors acknowledge funding from the Polish Ministry of Science via grant No. 2254/B/T02/2009/37.

\section{REFERENCES}

1. Deane,A., I. Kevrekidis, G. Karniadakis, and S. Orszag. 1991. Low-dimensional models for complex geometry flows: Application to grooved channels and circular cylinders. Phys. Fluids A 3:2337-54.

2. Morzyński, M., W. Stankiewicz, B. R. Noack, F. Thiele, and G. Tadmor. 2006. Generalized mean-field model with continuous mode interpolation for flow control. AIAA Paper No. 2006-3488.

3. Rossow, C., and N. Kroll. 2008. Opportunities for next generation product development. AIAA Paper No. 2008-0712.

4. Khibnik, A., S. Narayanan, C. Jacobson, and K. Lust. 2000. Analysis of low dimensional dynamics of flow separation. In: Continuation methods in fluid dynamics. Notes on numerical fluid mechanics. Vieweg. 64:167-78.

5. Ma, X., and G. Karniadakis. 2002. A low-dimensional model for simulating threedimensional cylinder flow. J. Fluid Mech. 458:181-90.

6. Jørgensen, B., J. Sørensen, and M. Brøns. 2003. Low-dimensional modeling of a driven cavity flow with two free parameters. Theor. Comput. Fluid Dyn. 16:299317.

7. Bergmann, M., L. Cordier, and J. P. Brancher. 2005. Optimal rotary control of the cylinder wake using proper orthogonal decomposition reduced order model. Phys. Fluids 17:097101-21.

8. Rowley, C. W. 2005. Model reduction for fluids using balanced proper orthogonal decomposition. Int. J. Bifurcation Chaos 15(3):997-1013.

9. Siegel, S., K. Cohen, J. Seigel, and T. McLaughlin. 2006. Proper orthogonal decomposition snapshot selection for state estimation of feedback controlled flows. AIAA Paper No. 2006-1400.

10. Rowley, C. W., I. Mezić, S. Bagheri, P. Schlatter, and D. S. Henningson. 2009. Spectral analysis of nonlinear flows. J. Fluid Mech. 645:115-27.

11. Holmes, P., J. L. Lumley, and G. Berkooz. 1998. Turbulence, coherent structures, dynamical systems and symmetry. Cambridge: Cambridge University Press.

12. Noack, B. R., P. Papas, and P. A. Monkewitz. 2005. The need for a pressure-term representation in empirical Galerkin models of incompressible shear-flows. J. Fluid Mech. 523:339-65.

13. Noack, B. R., M. Schlegel, B. Ahlborn, G. Mutschke, M. Morzyński, P. Comte, and G. Tadmor. 2008. A finite-time thermodynamics of unsteady fluid flows. J. NonEquilibr. Thermodyn. 33(2):103-48.

14. Abdessemed, N., S. Sherwin, and V. Theofilis. 2006. Linear stability of the flow past a low pressure turbine blade. 36th AIAA Fluid Dynamics Conference and Exhibit. 
15. Crouch, J., A. Garbaruk, and D. Magidov. 2007. Predicting the onset of flow unsteadiness based on global instability. J. Comput. Phys. 224(2):924-40.

16. Giannetti, F., and P. Luchini. 2007. Structural sensitivity of the first instability of the cylinder wake. J. Fluid Mech. 581:167-97.

17. Theofilis, V. 2003. Advances in global linear instability analysis of nonparallel and three-dimensional flows. Prog. Aerospace Sci. 39(4):249-315.

18. Morzyński, M., and F. Thiele. 2008. Finite element method for global stability analysis of 3D flows. AIAA Paper No. 2008-3865.

19. Noack, B. R., K. Afanasiev, M. Morzyński, G. Tadmor, and F. Thiele. 2003. A hierarchy of low-dimensional models for the transient and post-transient cylinder wake. J. Fluid Mech. 497:335-63.

20. Morzyński, M., K. Afanasiev, and F. Thiele. 1999. Solution of the eigenvalue problems resulting from global non-parallel flow stability analysis. Comput. Meth. Appl. Mech. Eng. 169:161-76.

21. Stankiewicz, W., M. Morzyński, B. R. Noack, and G. Tadmor. 2008. Reduced order Galerkin models of flow around NACA-0012 airfoil. Math. Modell. Anal. 13(1):11322 .

22. Sirovich, L. 1987. Turbulence and the dynamics of coherent structures. Quart. Appl. Math. 45:561-90. 